\title{
Lower bounds of solutions of the Magnetohydrodynamics Equations in Homogeneous Sobolev Spaces
}

\author{
Diego Marcon ${ }^{1}$ \\ Departamento de Matemática Pura e Aplicada, UFRGS, Porto Alegre, RS \\ Wilberclay G. Melo \\ Departamento de Matemática, UFS, São Cristovão,SE \\ Lineia Schütz ${ }^{3}$ \\ Departamento de Matemática Pura e Aplicada, UFRGS, Porto Alegre, RS \\ Juliana Sartori Ziebell ${ }^{4}$ \\ Instituto de Matemática, Estatística e Física, FURG, Santo Antônio da Patrulha, RS
}

Resume. In this paper, we obtain new lower bounds on the Homogeneous Sobolev-norms of the maximal solution of the Magnetohydrodynamics Equations. This gives us some insight on the blow-up behavior of the solution. We utilize standard techniques from the NavierStokes Equations.

Key words. Magnethohydrodinamics equations, blow-up criterion, lower bounds

\section{Introduction}

Magnetohydrodynamics (MHD) describes the motion of electrically conducting fluids, such as liquid metals, salt water, or plasmas, in the presence of magnetic fields. The MHD Equations form a system of equations that combine fluid dynamics with Maxwell's theory of electromagnetism. For an introduction on the subject, see [14]. In this paper, we consider the three-dimensional MHD Equations for incompressible flows:

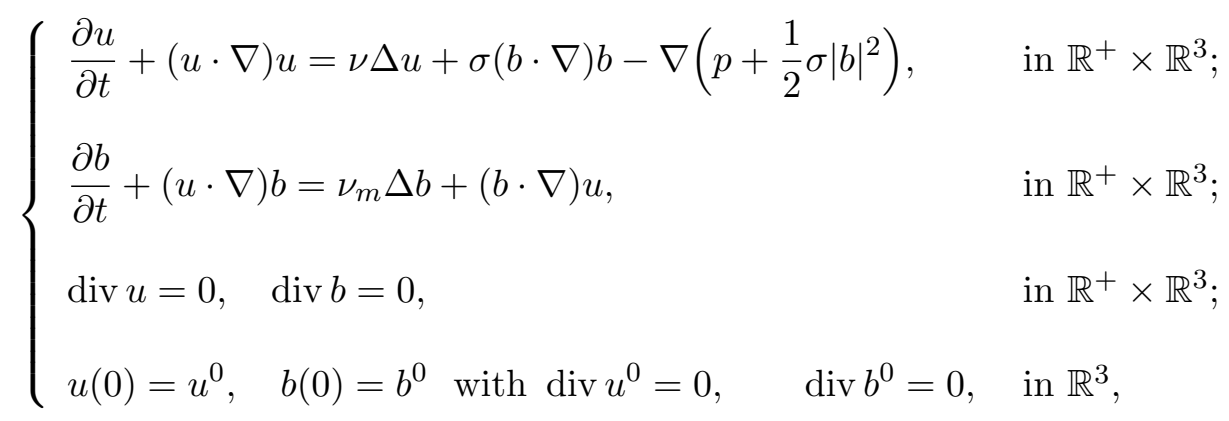

\footnotetext{
${ }^{1}$ diego.marcon@ufrgs.br

2 wilberclay@bol.com.br

${ }^{3}$ lineia.schutz@ufrgs.br

${ }^{4}$ julianaziebell@furg.br
} 
where $u(x, t)=\left(u_{1}(x, t), u_{2}(x, t), u_{3}(x, t)\right) \in \mathbb{R}^{3}$ denotes the velocity field of the fluid and $b(x, t)=\left(b_{1}(x, t), b_{2}(x, t), b_{3}(x, t)\right) \in \mathbb{R}^{3}$ the magnetic field. The remaining terms are the hydrostatic pressure $p(x, t) \in \mathbb{R}$, the initial data $u_{0}, b_{0}$ in $L^{2}\left(\mathbb{R}^{3} ; \mathbb{R}^{3}\right)$, and the positive constants $\nu, \nu_{m}$, and $\sigma$. These constants denote, respectively, the kinematic constant $\nu=1 / R_{e}$, the magnetic diffusivity constant $\nu_{m}=1 / R_{m}$, and $\sigma=M^{2} / R_{e} R_{m}$, where $R_{e}$ is the Reynolds number, $R_{m}$ is the magnetic Reynolds number, and $M$ is the Hartman number.

The existence and the uniqueness of solutions of MHD systems have been extensively studied $[2,4-6,12,13,15,17-19]$. Duvaut and Lions [5] introduced a class of weak solutions with finite energy and proved the existence and uniqueness of weak and strong solutions of the MHD problem. Sermange and Teman [15] obtained results of regularity for solution to the MHD system considering fluids in limited regions with appropriate boundary conditions, or fluids in $\mathbb{R}^{3}$ with periodicity conditions. The problem in limited region was also studied by Rojas and Boldrini [12] . They showed, by using Galerkin method, the existence of weak solutions to the MHD problem in two or three dimensions. In general, one can only ensure the existence of a maximal time $T^{*}>0$ for which the MHD system (1) has a classical solution $u(x, t), b(x, t)$ defined for $(t, x) \in\left[0, T^{*}\right) \times \mathbb{R}^{3}$. This is the analog of the corresponding Incompressible Navier-Stokes problem. In fact, in the absence of a magnetic field, the MHD Equations reduce to the Navier-Stokes Equations. For regularity results and blow-up estimates of the Navier-Stokes Equations, see [3,7-11,16].

For Homogeneous Sobolev Spaces $H^{s_{0}}\left(\mathbb{R}^{3}\right)$, Yuan [17] obtained the existence of strong solutions of $(1)$ in $\left[0, T^{*}\right) \times \mathbb{R}^{3}$. More precisely, for $s_{0}>3 / 2$ and for divergence-free $u^{0}, b^{0} \in H^{s_{0}}\left(\mathbb{R}^{3}\right)$, there exists a positive time $T^{*}>0$, depending on the initial conditions $u^{0}, b^{0}$, such that (1) admits a unique classical solution $(u, b)$, defined on $\left[0, T^{*}\right) \times \mathbb{R}^{3}$. Motivated by He and Xin [6], Yuan also provides blow-up criteria that is independent of the magnetic field $b$, see [17, Theorem 1.1].

In this paper, we suppose $T^{*}<\infty$ and we establish properties of the maximal solution of (1). Inspired by Benameur [3], we obtain blow-up estimates for strong solutions of the Incompressible MHD Equations in the Homogeneous Sobolev Space $\dot{H}^{s}\left(\mathbb{R}^{3}\right)$, for $s>1 / 2$.

Set $\lambda=\min \left\{\nu, \nu_{m}\right\}$ and $\kappa=\max \{1, \sigma\}^{-1}$. Our main theorem is the following:

Theorem 1.1. Fix $s_{0}>3 / 2$ and let $u^{0}, b^{0} \in H^{s_{0}}\left(\mathbb{R}^{3}\right)$ be such that $\operatorname{div} u^{0}=\operatorname{div} b^{0}=0$. Consider $u, b \in C\left(\left[0, T^{*}\right), H^{s}\left(\mathbb{R}^{3}\right)\right)$ the strong solution of $(1)$, defined in its maximal interval of definition $\left[0, T^{*}\right)$. If $T^{*}<+\infty$, then for every $\delta \in(0,1)$ and for every $s \geq$ $1 / 2+\delta$, we have

$$
\|(u, b)(t)\|_{\dot{H}^{s}\left(\mathbb{R}^{3}\right)}\|(u, b)(t)\|_{L^{2}\left(\mathbb{R}^{3}\right)}^{p(s)} \geq \frac{c(s, \delta, \sigma) \lambda^{q(s, \delta)}}{\left(T^{*}-t\right)^{r(s, \delta)}},
$$

where

$$
p(s, \delta):=\frac{2 s}{1+2 \delta}-1, \quad q(s, \delta):=\frac{(2-\delta) s}{1+2 \delta}, \quad \text { and } r(s, \delta):=\frac{s \delta}{1+2 \delta} .
$$

Remark 1.1. We observe that Theorem 1.1 extends and improves the results of [3]. If $b=0$, the MHD Equations become the Navier-Stokes Equations. In this case, the choice $\delta=1 / 2$ provides us with Benameur's estimate, see [3, Theorem 1.3, (1.2)]. Moreover, our approach allows us to consider any $s>3 / 2$ whereas in [3], the author assumes $s>5 / 2$. 
Remark 1.2. Now, we show how different choices of $\delta$ and $s$ can provide powerful estimates.

i. Follows from Theorem 1.1, by chossing $\delta=1 / 2$, that, for each $s \geq 1$,

$$
\|(u, b)(t)\|_{\dot{H}^{s}}\|(u, b)(t)\|_{L^{2}}^{s-1} \geq \frac{c(s, \sigma) \lambda^{3 s / 4}}{\left(T^{*}-t\right)^{s / 4}}, \text { for all } t \in\left[0, T^{*}\right) .
$$

ii. It suffices to take $s=1$ in remark (1.2.i.) to get a version of Leray's Inequality [9] for the MHD Equations

$$
\|(\nabla u, \nabla b)(t)\|_{L^{2}\left(\mathbb{R}^{3}\right)} \geq \frac{c \lambda^{3 / 4}}{\left(T^{*}-t\right)^{1 / 4}} \text { for all } t \in\left[0, T^{*}\right), T^{*}<\infty .
$$

iii. For each $\frac{1}{2}<s<\frac{3}{2}$,

$$
\|(u, b)(t)\|_{\dot{H}^{s}\left(\mathbb{R}^{3}\right)} \geq \frac{c(s, \sigma) \lambda^{\frac{5}{4}-\frac{s}{2}}}{\left(T^{*}-t\right)^{\frac{s}{2}-\frac{1}{4}}} \text { for all } t \in\left[0, T^{*}\right) .
$$

This is Theorem 1.1 with $s=\delta+1 / 2$.

Structure of the paper. In the next section, we fix the notation, and we state the results needed in the proof of Theorem 1.1. The section three is concerned with the proof of Theorem 1.1. In the section four, we present a brief conclusion of our work.

\section{Preliminaries}

Here, we introduce some notations and state the results that we use in the rest of the paper.

If $u=\left(u_{1}, u_{2}, u_{3}\right)$ and $v=\left(v_{1}, v_{2}, v_{3}\right)$ are vector fields, the tensor product $u \otimes v$ is $u \otimes v:=\left(v_{1} u, v_{2} u, v_{3} u\right)$ and its divergence is $\operatorname{div}(u \otimes v)=\left(\operatorname{div}\left(v_{1} u\right), \operatorname{div}\left(v_{2} u\right), \operatorname{div}\left(v_{3} u\right)\right)$.

The Fourier Transform of $f$ is given by $\mathcal{F}(f)(\xi)=\hat{f}(\xi):=\int_{\mathbb{R}^{3}} e^{-i \xi \cdot x} f(x) d x$. We consider the Euclidean norm $|z|^{2}=z \cdot z$ in $\mathbb{C}^{3}$.

For $s \in \mathbb{R}$, the Homogeneous Sobolev Space $\dot{H}^{s}\left(\mathbb{R}^{3}, \mathbb{R}^{3}\right)$ is the space of tempered distributions $f$ for which $\|f\|_{\dot{H}^{s}} \stackrel{\text { def }}{=} \sqrt{\int_{\mathbb{R}^{3}}|\xi|^{2 s}|\hat{f}(\xi)|^{2} d \xi}<+\infty$. In this way, we note that if $u, b \in \dot{H}^{s}\left(\mathbb{R}^{3}, \mathbb{R}^{3}\right)$, we have $\|(u, b)\|_{\dot{H}^{s}}=\sqrt{\|u\|_{\dot{H}^{s}}^{2}+\|b\|_{\dot{H}^{s}}^{2}}$. The inner product in given by $\langle f, g\rangle_{\dot{H}^{s}} \stackrel{\text { def }}{=} \int_{\mathbb{R}^{3}}|\xi|^{2 s} \hat{f}(\xi) \cdot \hat{g}(\xi) d \xi$, where $\hat{f} \cdot \hat{g}=\sum_{i=1}^{3} \hat{f}_{i} \overline{\hat{g}}_{i}$. For Homogeneous Sobolev Spaces, we refer to the book [1].

For instance, the following basics interpolations inequalities holds:

Lemma 2.1. For $0<s_{0} \leq s$, the space $L^{2} \cap \dot{H}^{s}$ is a subset of $\dot{H}^{s_{0}}$, and we have

$$
\|f\|_{\dot{H}^{s_{0}}} \leq\|f\|_{L^{2}}^{1-s_{0} / s}\|f\|_{\dot{H}^{s}}^{s_{0} s} .
$$


Proof. This is a particular case of [1, Proposition 1.32].

Lemma 2.2. Let $\theta$ be a tempered distribution. Then, for $0<\delta<1$ and $s \geq 1 / 2+\delta$, we have

$$
\|\theta\|_{\dot{H}^{s+1-\delta}} \leq\|\theta\|_{\dot{H}^{s}}^{\delta}\|\nabla \theta\|_{\dot{H}^{s}}^{1-\delta}
$$

Proof. Since $\widehat{\nabla \theta}(\xi)=i \xi \hat{\theta}(\xi)$, the proof is a simple application of Hölder's inequality

Next, we state a version of what is known as Chemin's Lemma:

Lemma 2.3. Fix $\delta \in(0,1)$, let $\eta=1 / 2+\delta<3 / 2$ and $\eta^{\prime}=s+1-\delta<3 / 2$. Then there exists a constant $C(s, \delta)>0$ such that, for every $f, g \in \dot{H}^{\eta}\left(\mathbb{R}^{3}\right) \cap \dot{H}^{\eta^{\prime}}\left(\mathbb{R}^{3}\right)$,

$$
\|f g\|_{\dot{H}^{s}} \leq C(s, \delta)\|f\|_{\dot{H}^{\eta}}\|g\|_{\dot{H}^{\eta^{\prime}}} .
$$

Proof. Observe $\eta+\eta^{\prime}=s+3 / 2$ and apply Chemin's Lemma [3, Lemma 3.1].

\section{Proof of Theorem 1.1}

In this section, we prove Theorem 1.1.

Proof. Since $\operatorname{div} u=\operatorname{div} b=0$, by taking the $\dot{H}^{s}$-scalar product of the first equation in (1) with $u$, we obtain

$$
\frac{1}{2} \partial_{t}\|u\|_{\dot{H}^{s}}^{2}+\nu\|\nabla u\|_{\dot{H}^{s}}^{2}-\operatorname{Re}\left[\langle u \otimes u, \nabla u\rangle_{\dot{H}^{s}}\right]+\operatorname{Re}\left[\sigma\langle b \otimes b, \nabla u\rangle_{\dot{H}^{s}}\right]=0,
$$

where $R e[z]$ denotes the real part of the complex number $z$. Then,

$$
\begin{aligned}
\frac{1}{2} \partial_{t}\|u\|_{\dot{H}^{s}}^{2}+\nu\|\nabla u\|_{\dot{H}^{s}}^{2} & =\operatorname{Re}\left[\langle u \otimes u-\sigma(b \otimes b), \nabla u\rangle_{\dot{H}^{s}}\right] \\
& \leq\|u \otimes u\|_{\dot{H}^{s}}\|\nabla u\|_{\dot{H}^{s}}+\sigma\|b \otimes b\|_{\dot{H}^{s}}\|\nabla u\|_{\dot{H}^{s}} .
\end{aligned}
$$

Now, we apply Lemma 2.3 and Lemma 2.2 to the right hand side of (7) to obtain

$$
\begin{aligned}
\frac{1}{2} \partial_{t}\|u\|_{\dot{H}^{s}}^{2} & +\nu\|\nabla u\|_{\dot{H}^{s}}^{2} \leq c(s, \delta)\left[\|u\|_{\dot{H}^{1 / 2+\delta}}\|u\|_{\dot{H}^{s+1-\delta}}+\sigma\|b\|_{\dot{H}^{1 / 2}+\delta}\|b\|_{\dot{H}^{s+1-\delta}}\right]\|\nabla u\|_{\dot{H}^{s}} \\
& \leq c(s, \delta, \sigma)\left[\|u\|_{\dot{H}^{1 / 2+\delta}}\|u\|_{\dot{H}^{s}}^{\delta}\|\nabla u\|_{\dot{H}^{s}}^{2-\delta}+\|b\|_{\dot{H}^{1 / 2+\delta}}\|b\|_{\dot{H}^{s}}^{\delta}\|\nabla b\|_{\dot{H}^{s}}^{1-\delta}\|\nabla u\|_{\dot{H}^{s}}\right] .
\end{aligned}
$$

Thus, Young's Inequality implies

$\partial_{t}\|u\|_{\dot{H}^{s}}^{2}+\nu\|\nabla u\|_{\dot{H}^{s}}^{2} \leq c(s, \delta, \sigma)\left[\nu^{-\frac{2-\delta}{\delta}}\|u\|_{\dot{H}^{1 / 2+\delta}}^{2 / \delta}\|u\|_{\dot{H}^{s}}^{2}+\nu^{-1}\|b\|_{\dot{H}^{1 / 2+\delta}}^{2}\|b\|_{\dot{H}^{s}}^{2 \delta}\|\nabla b\|_{\dot{H}^{s}}^{2-2 \delta}\right]$.

From the second equation of the system (1), we obtain an analogous estimate:

$$
\partial_{t}\|b\|_{\dot{H}^{s}}^{2}+\nu_{m}\|\nabla b\|_{\dot{H}^{s}}^{2} \leq c(s, \delta)\left[\nu_{m}^{-1}\|b\|_{\dot{H}^{1 / 2+\delta}}^{2}\|u\|_{\dot{H}^{s}}^{2 \delta}\|\nabla u\|_{\dot{H}^{s}}^{2-2 \delta}+\nu_{m}^{-\frac{2-\delta}{\delta}}\|u\|_{\dot{H}^{1 / 2+\delta}}^{2 / \delta}\|b\|_{\dot{H}^{s}}^{2}\right]
$$


Now, we set $\lambda=\min \left\{\nu, \nu_{m}\right\}$ and add (8) and (9). We obtain

$$
\begin{aligned}
\partial_{t}\|(u, b)\|_{\dot{H}^{s}}^{2}+ & \lambda\|(\nabla u, \nabla b)\|_{\dot{H}^{s}}^{2} \leq c(s, \delta, \sigma)\left[\lambda^{-\frac{2-\delta}{\delta}}\|u\|_{\dot{H}^{1 / 2+\delta}}^{2 / \delta}\left(\|u\|_{\dot{H}^{s}}^{2}+\|b\|_{\dot{H}^{s}}^{2}\right)\right. \\
& \left.+\lambda^{-1}\|b\|_{\dot{H}^{1 / 2+\delta}}^{2}\|b\|_{\dot{H}^{s}}^{2 \delta}\|\nabla b\|_{\dot{H}^{s}}^{2-2 \delta}+\lambda^{-1}\|b\|_{\dot{H}^{1 / 2+\delta}}^{2}\|u\|_{\dot{H}^{s}}^{2 \delta}\|\nabla u\|_{\dot{H}^{s}}^{2-2 \delta}\right] .
\end{aligned}
$$

By Young's inequality again, it follows that

$$
\partial_{t}\|(u, b)\|_{\dot{H}^{s}}^{2}+\frac{\lambda}{2}\|(\nabla u, \nabla b)\|_{\dot{H}^{s}}^{2} \leq c(s, \delta, \sigma) \lambda^{-\frac{2-\delta}{\delta}}\left[\|u\|_{\dot{H}^{1 / 2+\delta}}^{2 / \delta}+\|b\|_{\dot{H}^{1 / 2+\delta}}^{2 / \delta}\right]\|(u, b)\|_{\dot{H}^{s}}^{2} .
$$

In particular,

$$
\partial_{t}\|(u, b)\|_{\dot{H}^{s}}^{2} \leq c(s, \delta, \sigma) \lambda^{-\frac{2-\delta}{\delta}}\left[\|u\|_{\dot{H}^{1 / 2+\delta}}^{2 / \delta}+\|b\|_{\dot{H}^{1 / 2+\delta}}^{2 / \delta}\right]\|(u, b)\|_{\dot{H}^{s}}^{2} .
$$

Thus, by Gronwall's Inequality with $0 \leq a \leq t<T^{*}$,

$$
\|(u, b)(t)\|_{\dot{H}^{s}}^{2} \leq\|(u, b)(a)\|_{\dot{H}^{s}}^{2} \exp \left(c(s, \delta, \sigma) \lambda^{-\frac{2-\delta}{\delta}} \int_{a}^{t}\left[\|u(\tau)\|_{\dot{H}^{1 / 2+\delta}}^{2 / \delta}+\|b(\tau)\|_{\dot{H}^{1 / 2+\delta}}^{2 / \delta}\right] d \tau\right) .
$$

Set $\theta:=(1+2 \delta) / 2 s$. By Lemma 2.1, whenever $s \geq 1 / 2+\delta$ then

$$
\|f\|_{\dot{H}^{1 / 2+\delta}} \leq\|f\|_{L^{2}}^{1-\theta}\|f\|_{\dot{H}^{s}}^{\theta} .
$$

Estimate (11), applied to $u$ and $b$, yields

$$
\begin{aligned}
\|u\|_{\dot{H}^{1 / 2+\delta}}^{2 / \delta}+\|b\|_{\dot{H}^{1 / 2+\delta}}^{2 / \delta} & \leq 2^{1 / \delta}\left(\|u\|_{\dot{H}^{1 / 2+\delta}}^{2}+\|b\|_{\dot{H}^{1 / 2+\delta}}^{2}\right)^{1 / \delta} \\
& \leq 2^{1 / \delta}\left[\left(\|u\|_{L^{2}}^{2-2 \theta}+\|b\|_{L^{2}}^{2-2 \theta}\right)\|u\|_{\dot{H}^{s}}^{2 \theta}+\left(\|u\|_{L^{2}}^{2-2 \theta}+\|b\|_{L^{2}}^{2-2 \theta}\right)\|b\|_{\dot{H}^{s}}^{2 \theta}\right]^{1 / \delta} \\
& \leq 2^{1 / \delta}\left[\|u\|_{L^{2}}^{2}+\|b\|_{L^{2}}^{2}\right]^{(1-\theta) / \delta}\left[\|u\|_{\dot{H}^{s}}^{2}+\|b\|_{\dot{H}^{s}}^{2}\right]^{\theta / \delta}
\end{aligned}
$$

This and (10) imply

$$
\begin{aligned}
\|u(t)\|_{\dot{H}^{1 / 2+\delta}}^{2 / \delta}+\|b(t)\|_{\dot{H}^{1 / 2+\delta}}^{2 / \delta} \leq & 2^{1 / \delta}\|(u, b)(t)\|_{L^{2}}^{(2-2 \theta) / \delta}\|(u, b)(a)\|_{\dot{H}^{s}}^{2 \theta / \delta} \times \\
& \times \exp \left(c(s, \delta, \sigma) \lambda^{-\frac{2-\delta}{\delta}} \int_{a}^{t}\left[\|u(\tau)\|_{\dot{H}^{1 / 2+\delta}}^{2 / \delta}+\|b(\tau)\|_{\dot{H}^{1 / 2+\delta}}^{2 / \delta}\right] d \tau\right)
\end{aligned}
$$

Then, by using the decay of the $L^{2}-$ norm, we obtain

$$
f(t) \exp \left(-c(s, \delta, \sigma) \lambda^{-\frac{2-\delta}{\delta}} \int_{a}^{t} f(\tau) d \tau\right) \leq 2^{1 / \delta}\|(u, b)(a)\|_{L^{2}}^{(2-2 \theta) / \delta}\|(u, b)(a)\|_{\dot{H}^{s}}^{2 \theta / \delta}
$$

where

$$
f(t):=\|u(t)\|_{\dot{H}^{1 / 2+\delta}}^{2 / \delta}+\|b(t)\|_{\dot{H}^{1 / 2+\delta}}^{2 / \delta}
$$


Now, we integrate on $[a, T]$, with $T<T^{*}$ :

$$
\begin{aligned}
& 1-\exp \left(-c(s, \delta, \sigma) \lambda^{-\frac{2-\delta}{\delta}} \int_{a}^{T} f(\tau) d \tau\right) \leq \\
& \quad c(s, \delta, \sigma) \lambda^{-\frac{2-\delta}{\delta}}\|(u, b)(a)\|_{L^{2}}^{(2-2 \theta) / \delta}\|(u, b)(a)\|_{\dot{H}^{s}}^{2 \theta / \delta}(T-a) .
\end{aligned}
$$

Let $T \rightarrow T^{*}$ and use (10):

$$
1 \leq c(s, \delta, \sigma) \lambda^{-\frac{2-\delta}{\delta}}\|(u, b)(a)\|_{L^{2}}^{(2-2 \theta) / \delta}\|(u, b)(a)\|_{\dot{H}^{s}}^{2 \theta / \delta}\left(T^{*}-a\right) .
$$

Hence,

$$
\|(u, b)(a)\|_{\dot{H}^{s}}\|(u, b)(a)\|_{L^{2}}^{p(s, \delta)} \geq \frac{c(s, \delta, \sigma) \lambda^{q(s, \delta)}}{\left(T^{*}-a\right)^{r(s, \delta)}} .
$$

\section{Conclusions}

In this work, we derived new lower bounds on the Homogeneous Sobolev-norms of the maximal solution of the Magnetohydrodynamics Equations. Our main result is present in Theorem 1.1. This estimate give us some insight on the blow-up behavior of the solution.

Acknowledgements. We thank Prof. Paulo Zíngano for comments and fruitful discussions. D. Marcon was partially supported by $\mathrm{CNPq}-$ Brazil through the postdoctoral scholarship PDJ/501839/2013-5. W. Melo was partially supported by CAPES (Ciência sem Fronteiras) grant 2778-13-0. W. Melo would like to thank the Federal University of Sergipe for supporting his long term visit to the University of New Mexico during the academic year 2013-2014.

\section{References}

[1] H. Bahouri, J.-Y. Chemin, and R. Danchin. Fourier analysis and nonlinear partial differential equations, volume 343 of Grundlehren der Mathematischen Wissenschaften [Fundamental Principles of Mathematical Sciences]. Springer, Heidelberg, 2011.

[2] J. T. Beale, T. Kato, and A. Majda. Remarks on the breakdown of smooth solutions for the 3-D Euler equations. Communications in Mathematical Physics, 94(1):61-66, 1984.

[3] J. Benameur. On the blow-up criterion of 3D Navier-Stokes equations. Journal of Mathematical Analysis and Applications, 371(2):719-727, 2010.

[4] R. E. Caflisch, I. Klapper, and G. Steele. Remarks on singularities, dimension and energy dissipation for ideal hydrodynamics and MHD. Communications in Mathematical Physics, 184(2):443-455, 1997. 
[5] G. Duvaut and J.-L. Lions. Inéquations en thermoélasticité et magnétohydrodynamique. Archive for Rational Mechanics and Analysis, 46:241-279, 1972.

[6] C. He and Z. Xin. On the regularity of weak solutions to the magnetohydrodynamic equations. Journal of Differential Equations, 213(2):235-254, 2005.

[7] T. Kato. Strong $L^{p}$-solutions of the Navier-Stokes equation in $\mathbb{R}^{m}$, with applications to weak solutions. Mathematische Zeitschrift, 187(4):471-480, 1984.

[8] H.-O. Kreiss, T. Hagstrom, J. Lorenz, and P. Zingano. Decay in time of incompressible flows. Journal of Mathematical Fluid Mechanics, 5(3):231-244, 2003.

[9] J. Leray. Sur le mouvement d'un liquide visqueux emplissant l'espace. Acta Mathematica, 63(1):193-248, 1934.

[10] K. Masuda. Weak solutions of navier-stokes equations. Tohoku Mathematical Journal, 36(4):623-646, 1984.

[11] J. C. Robinson, W. Sadowisk, and R. P. Silva. Lower bounds on blow up solutions of the three-dimensional Navier-Stokes equations in homogeneous Sobolev spaces. Journal of Mathematical Physics, 53(11): 115618, 2012.

[12] M. A. Rojas-Medar, J. L. Boldrini. Magneto-micropolar fuid motion: existence of weak solutions. Revista Matemática Complutence, 11(2): 443-460, 1998.

[13] M. E. Schonbek, T. P. Schonbek, and E. Süli. Large-time behaviour of solutions to the magnetohydrodynamics equations. Mathematische Annalen, 304(4):717-756, 1996.

[14] D. D. Schnack. Lectures in Magnetohydrodynamics: With an Appendix on Extended MHD, Lecture Notes in Physics 780. Springer, Berlin Heidelberg, 2009.

[15] M. Sermange and R. Temam. Some mathematical questions related to the MHD equations. Communications on Pure and Applied Mathematics, 36(5):635-664, 1983.

[16] M. Wiegner. Decay results for weak solutions of the Navier-Stokes equations on $\mathbb{R}^{n}$. Journal of the London Mathematical Society. Second Series, 35(2):303-313, 1987.

[17] J. Yuan. Existence theorem and blow-up criterion of the strong solutions to the magneto-micropolar fluid equations. Mathematical Methods in the Applied Sciences, 31(9):1113-1130, 2008.

[18] J. Yuan. Existence theorem and regularity criteria for the generalized MHD equations. Nonlinear Analysis. Real World Applications. An International Multidisciplinary Journal, 11(3):1640-1649, 2010.

[19] Y. Zhou. Remarks on regularities for the 3D MHD equations. Discrete and Continuous Dynamical Systems. Series A, 12(5):881-886, 2005. 